\title{
Performance of SO Bioline FK80 test kit in diagnosis of malaria at Adama Malaria Center, southeast Oromia, Ethiopia
}

\author{
Sena Bayisa ${ }^{1}$, Tadesse Kebede ${ }^{2}$, Gessessew Bugssa ${ }^{1}$, Megbaru Alemu ${ }^{1}$ \\ ${ }^{1}$ Institute of Biomedical Sciences, College of Health Science, Mekele University, Mekele, Ethiopia \\ ${ }^{2}$ Department of Microbiology, Immunology and Parasitology (DMIP), School of Medicine, Addis Ababa University, Addis Ababa, Ethiopia
}

Email address:

sena.bayisagesp@gmail.com (S. Bayissa), tadesse.kebedeg@gmail.com (T. kebede), bugssag@gmail.com (G. Bugssa), megibgesep@gmail.com (M. Alemu)

\section{To cite this article:}

Sena Bayisa, Tadesse Kebede, Gessessew Bugssa, Megbaru Alemu. Performance of SO Bioline FK80 Test Kit in Diagnosis of Malaria at Adama Malaria Center, Southeast Oromia, Ethiopia. Science Journal of Public Health. Vol. 3, No. 1, 2015, pp. 31-36.

doi: $10.11648 /$ j.sjph.20150301.16

\begin{abstract}
Background: The use of RDT (Rapid diagnostic tests) for malaria offers the potential to extend accurate malaria diagnosis to areas where microscopy services are not available. Objective: To evaluate the performance of SD FK80 test kit for the diagnosis of malaria and assess the trend of malaria transmission in Adama Malaria Center, southeast Ethiopia. Methods: A cross sectional study was conducted to evaluate the performance of SD FK80 kit for malaria (P.falciparum/P.vavix) diagnosis November to December 2012 at Adama Malaria Center, Southeastern Oromia. Three hundred eighty four blood samples were collected from febrile patients attending the outpatient department of Adama Malaria Center during the study period. The blood samples were analyzed with microscopy and RDT (SD Bioline P.falciparum /P.vivax) for the detection and identification of Plasmodium parasites. The data was entered into Microsoft ${ }^{\circledR}$ Excel and was transported to SPSS version 17.0 for statistical analysis. Sensitivity, specificity, positive and negative predictive values of the tests was calculated using microscopy as the reference standard. Result: Among the examined individuals, 107(27.9\%) were found to be positive for plasmodium infection of which $23.7 \%$ were infected with P.vavix, and 3.9\% were infected with P.falciparum while $0.3 \%$ were mixed infections The sensitivity, specificity, positive predictive value and negative predictive values of the SD Bioline were 90.7\%, 96\%, 89.8\%, and $96.4 \%$, respectively taking blood film as a gold standard. SD Bioline FK80 P.falciparum/P.vivax was performed satisfactorily for the diagnosis of P.falciparum and P.vivax infections. Conclusion: Based on our finding, Rapid test kit is satisfactory for diagnosis of P.falciparum and P.vivax infections. It is a useful adjunct to microscopy especially in our country where there is limited man power and resource.
\end{abstract}

Keywords: Malaria, Microscopy, RDT, P.falciparum, P.vivax

\section{Introduction}

Malaria is a major cause of morbidity and mortality. In 2006, about 3.3 billion persons were at risk of malaria infection of which 1.2 billion were at higher risk, mostly in Africa [1]. About $68 \%$ of the Ethiopia's population is at risk of malaria, representing approximately 52 million people [2].

In Ethiopia, malaria transmission is generally unstable and seasonal, the level of transmission varying from place to place because of altitude, rainfall, temperature, and humidity. Unstable malaria occurs in most parts of the country particularly in the highland fringes where climatic conditions are conducive for its transmission [3]. Major transmission of malaria occurs between September to December while minor transmission occurs during April and May [4]. Some localities experience perennial malaria, due to environmental and climatological situations that permit the continual breeding of vectors. Plasmodium falciparum and P.vivax are the most dominant malaria parasites in the country, accounting for $60 \%$ and $40 \%$ of malaria cases respectively. P.malariae and P. ovale accounts for less than 1\% [5].

One of the most pronounced problems in controlling the morbidity and mortality caused by malaria is the limited access to effective diagnosis and treatment in areas where 
malaria is endemic. In many developing countries, microscopy is scarce. Even if microscopy is available the microscopists are insufficiently trained, supervised and are overloaded; the microscopes and reagents are of poor quality, and often the supply of electricity is unreliable [6]. Complete knowledge of the morphological features of the different blood stages of the different Plasmodia species is also difficult. These diagnostic limitations affect medical care providers, as malaria is a potentially fatal disease [7]. As a result, the World Health Organization has recognized the urgent need for simple and cost effective diagnostic tests for malaria to overcome the deficiencies of both light microscopy and clinical diagnosis [6]. Therefore, using highly sensitive and specific means of diagnosis for malaria is necessary to overcome the existing problem.

The use of RDT for malaria offers the potential to extend accurate malaria diagnosis to areas where microscopy services are not available such as in remote locations or after regular laboratory hours. Rapid malaria diagnostic tests have been developed in the lateral flow format. These tests use finger-stick or venous blood, take only 10 to 15 minutes, and do not require any laboratory equipment [8]. The use of this RDT may decrease the amount of time that it takes to determine that a patient is infected with malaria [9]. The SD FK80 Malaria Antigen Rapid Test (Standard Diagnostics, Hagal-Dong, Republic of Korea), further referred to as FK80, is a three-band RDT that targets HRP-2 and Pv-pLDH is target RDT used in this study. This kit is used in areas where both P.falciparum and P.vivax are prevalent; this combination has the advantage to distinguish unequivocally between the two species [10].

Diagnosis of malaria is still challenging in resource poor countries including Ethiopia. Skill of laboratory personnel is also another problem in malaria diagnosis. Parasitological confirmation of malaria through quality assured diagnosis in all settings before treatment should be given either by blood film or RDT. Timely confirmation of malaria can be achieved through good quality microscopy or Rapid Diagnostic Tests (RDTs) detecting P.falciparum and P.vivax which are more important species in our country. However, microscopy is not available in most peripheral areas of the country so there is a need for the use RDTs in such areas. This study provides basic information on the performance of malaria RDT (SD FK80) for the diagnosis of malaria. Furthermore, this study is aimed at assessing the prevalence of malaria among acute febrile patients attending Adama Malaria Center.

\section{Materials and Methods}

\subsection{Study Design}

Cross sectional study design was used.

\subsection{Study Area and Period}

The study was conducted at Adama Malaria Center which is located about $99 \mathrm{~km}$ from Addis Ababa in the southeast direction. The study was conducted during malaria incidence increasing season from mid November to December, 2012.This Malaria center is administered under the Adama Health Center.

Source population: All febrile patients attending the out patient department (OPD) of Adama Malaria Center during the study period.

Study Population: Acute febrile patients attending Adama Malaria Center seeking treatment were our study population.

\subsection{Inclusion and Exclusion Criteria}

Inclusion criteria: All febrile patients attending malaria center with fever or recent history of fever were included in the study.

Exclusion criteria: Those patients who refused to participate and who took malaria treatment with in the previous 5 days including the day of data collection were excluded from the study.

\subsection{Sample Size and Sampling Technique}

The sample size for this study was calculated based on the prevalence rate $(31 \%)$ of malaria reported from the previous study conducted somewhere in the country using RDT (Paracheck-pf ${ }^{\circledR}$ Test) [11], and taking 95\% confidence interval and 5\% marginal error, sample size (n) was determined using the single proportion formula ( $(n=z 2 p$ (1$\mathrm{p}) / \mathrm{d} 2$, where $\mathrm{n}=$ sample size, $\mathrm{z}=\mathrm{z}$ statistic for a level of confidence $(\mathrm{z}=1.96$ at $95 \% \mathrm{CI}), \mathrm{p}=$ expected Prevalence or proportion $(\mathrm{p}=0.31), \mathrm{d}=$ precision (if $5 \%, \mathrm{~d}=0.05$ ). Considering $16 \%$ non response rate, the final sample size was calculated to be 384 .

Sampling Technique: Non probability, convenience sampling method was used to select the study participants.

\subsection{Data Collection and Laboratory Diagnosis}

Sociodemographic data was collected from the study participants. Besides, blood was also collected from each volunteer participant.

Microscopic examination: blood was collected from the middle finger using a disposable blade after cleaning with an alcohol swab and dried with a piece of dry cotton. Then after wipping of the first blood drop, thick and thin films were made on the same slide, air-dried in a horizontal position and were placed in a slide box. Before staining, thin blood films were fixed in methanol for $30 \mathrm{sec}$. Then smears were stained with 3\% giemsa solution for 30 minutes according to WHO protocol [12]. Parasitemia and species was determined from thick and thin smear [13], respectively. The presence of gametocytes were also recorded. Microscopic examination of thick films using high power magnification for the presence of parasites and parasite species identification using thin films under 1000x oil immersion objective was done. Then the slides were cleaned with xylene and get dried and kept in a slide box for second round confirmatory microscopic examination and parasite density determination.

A minimum of 100 consecutive fields were counted in the thick blood film before a slide was classified as negative [9]. 
Recognized way of estimating the number of parasites present in $1 \mu \mathrm{l}$ of blood is to use a standard value for the WBC count $(8,000 \mathrm{WBC} / \mu \mathrm{l})$. After counting the number of parasites present until $200 \mathrm{WBC}$ has been seen, it was then multiplied by a factor of 40 to get the total parasite count per microliter of blood [14]. Densities (parasite per microliter of whole blood) were calculated and case definition was identified depending on parasite density : $\geq 10000=$ severe malaria case, $<10000=$ mild malaria case.

RDT examination: The same finger prick blood sample was used for carrying out RDT in parallel [SD BIOLIN Malaria Ag P.falciparum/P.vivax POCT] following the manufacturer's instructions (Standard Diagnostics of Korea). The FK80 kit is a lateral flow immunochromatographic RDT in a cassette format. Three lines are present, a control line which indicates whether the test is valid, a HRP-2 line and a Pv-pLDH line. According to the manufacturer's (Standard Diagnostics of Korea) instructions, a single HRP-2 line indicates an infection with P.falciparum, a single Pv-pLDH line indicates an infection with P.vivax and a combination of a HRP-2 line and a Pv-pLDH line indicates a mixed infection with P.falciparum and P.vivax. The other Plasmodium species cannot be detected with this test. For this evaluation, test kits of lot number RDT 145021 were used. Accordingly, $5 \mu 1$ of blood were taken by means of plastic sample loop and then 4 drops of buffer were added then read after 15 minutes and record at the correct time.

Three laboratory technicians were involved in the diagnosis of malaria during this study. Two microscopists who were blind to the results of RDTs and to each other examined the blood films. The K (kappa) value was determined for concordance of the two readers and interpreted as Poor agreement $\mathrm{k}(<0.20)$, Fair agreement $(0.20$ to 0.40$)$, Moderate agreement ( 0.40 to 0.60$)$, Good agreement $(0.60$ to 0.80$)$ and Very good agreement was taken to be $(0.80$ to 1.00 ) [15]. Besides, the third technician who was blind to microscopy result did the RDT test.

Discrepancies between Blood Slide (BS) readings and RDT results are important because of the fact that the two tests detect different components (whole parasites for microscopy and antigens for RDT). Accordingly, four categories of malaria status were defined: negative (BS negative and RDT negative); definite malaria (BS positive and RDT positive); probable malaria (BS positive and RDT negative); and possible malaria (RDT positive and BS negative).

Quality control: Clean, scratch free and new slides were used. The thickness of blood film was monitored, each time a smear is made. Quality of giemsa stain and $\mathrm{pH}$ (7.2) of giemsa stain were checked. Cleaned staining jar was used. Quality of package desiccant was also checked before use. Fresh blood samples were transferred directly to the sample pad by the provided sample applicator.

Data processing and analysis: The data was entered into Microsoff ${ }^{\circledR}$ Excel and was transported to SPSS version 17.0 for statistical analysis. Sensitivity, specificity, positive and negative predictive values of the tests was calculated using microscopy as the reference standard. The data is presented using descriptive statistics. Differences presenting $\mathrm{P}<0.05$ were considered as statistically significant. Agreements for 1 st and second readers were calculated by kappa value.

Ethical consideration: Ethical clearances were obtained from Addis Ababa University, College of Health Sciences, Department of Microbiology, Immunology and Parasitology. Further permission was obtained from Adama Health Center. Detailed information about the study was communicated to all subjects by the local language they can fully understand and participation was purely voluntary. Only individuals who gave oral/written consent were included in the study. Besides, positive/malaria cases were treated with appropriate antimalaria drugs (Chloroquine and Coartem).

\section{Results}

Socio demographic data: A total of 384 study participants (febrile patients) were included in the study from November to December 2102. Of the 384 study participants, $60.9 \%$ were males and $39.1 \%$ were females. The mean ages of the study participants were 25.5 years, with standard deviation of 16.15 .

Microscopy result: Blood film slides were examined with very good concordance between first and second $(\kappa=1.00)$. However, disconcordant result was confirmed by third reader. Among examined individuals 107 (27.9\%) were found to be positive for plasmodium infection of which $23.7 \%$ were infected with P.vavix, and $3.9 \%$ were infected with P.falciparum while $0.3 \%$ were mixed infections (Table 1). Among P.falciparum positive slides, $20 \%$ of the slides were positive for gametocyte.

Table 1. Prevalence of malaria parasite in relation to age by using microscopy at Adama Malaria Center from November to December 2012.

\begin{tabular}{|c|c|c|c|c|c|c|}
\hline \multirow{2}{*}{ Age group } & \multicolumn{4}{|c|}{ Malaria positive } & \multirow{2}{*}{ Total Negative (\%) } & \multirow{2}{*}{ Total examined } \\
\hline & PV N(\%) & PF N(\%) & Mixed & Total positive N (\%) & & \\
\hline$\leq 5$ & $7(21.9)$ & $2(6.3)$ & - & $9(28.2)$ & $23(72.9)$ & 32 \\
\hline $6-7$ & $31(32.3)$ & $4(4.2)$ & - & $35(36.5)$ & $61(63.5)$ & 96 \\
\hline$>17$ & $53(20.7)$ & $9(3.5)$ & $1(0.39)$ & $63(24.6)$ & $193(75.4)$ & 256 \\
\hline Total & 91(23.7) & $15(3.9)$ & $1(0.3)$ & $107(27.9)$ & $277(72.1)$ & 384 \\
\hline
\end{tabular}

Note: PV= P.vivax PF: P.falciparum Mixed: case infected with P.vivax and P.falciparum

$\mathrm{N}=$ frequency, $\%=$ percent 


\subsection{Performance of SD FK80}

Using Rapid Diagnostic Test (SD FK80), 108 (28.1\%) patients were found to be malaria positive, out of which $23.4 \%$ were infected with P.vivax, 2.9\% P.falciparum and
$1.8 \%$ were mixed infections. Among positive cases P.vivax, P.falciparum and mixed infection were $83.3 \%, 10.2 \%$ and $6.5 \%$, respectively (Table 2 ).

Table 2. Prevalence of malaria parasite in relation to age by using SD FK80 at Adama Malaria Center from November to December 2012.

\begin{tabular}{|c|c|c|c|c|c|c|}
\hline \multirow{2}{*}{ Age Group } & \multicolumn{4}{|c|}{ Malaria positive } & \multirow{2}{*}{ Negative n (\%) } & \multirow{2}{*}{ Total } \\
\hline & PV N (\%) & PF N (\%) & mixed & Total n (\%) & & \\
\hline$\leq 5$ & $9(28.1)$ & $2(6.3)$ & - & $11(34.4)$ & $21(68.7)$ & 32 \\
\hline $6-17$ & $28(29.2)$ & $5(5.2)$ & - & $33(34.4)$ & $63(65.6)$ & 96 \\
\hline$>17$ & $53(20.7)$ & $4(1.6)$ & $7(2.7)$ & $64(25)$ & $192(75)$ & 256 \\
\hline Total & $90(23.4)$ & $11(2.9)$ & $7(1.8)$ & $108(28.1)$ & $276(71.9)$ & 384 \\
\hline
\end{tabular}

Note: PV= P.vivax PF: P. falciparum Mixed: cases infected with P.vivax and P.falciparum

$\mathrm{N}=$ frequency, $\%=$ percent

Overall, the sensitivity and specificity of SD FK80 were found to be $90.7 \%$ and $96.7 \%$ respectively to diagnose both P.falciparum and P.vivax using blood film as a golden standard. Positive predictive value and Negative predictive value was $91.7 \%$ and $96.4 \%$, respectively. As a result, a conservative criterion, with a combination of blood slide and RDT, is needed to interpret the result in the study participants. Accordingly, $30.7 \%$ of the study participants were positive for malaria of which definite malaria, probable malaria, and possible malaria were $97 \%, 11 \%$ and $10 \%$, respectively (Table 3).

Table 3. Performance of SD FK80 taking microscopy as gold standard at Adama Malaria Center from November to December 2012.

\begin{tabular}{cccc}
\hline \multirow{2}{*}{ RDT result } & \multicolumn{2}{c}{ Blood film result } & \multirow{2}{*}{ Total } \\
\cline { 2 - 3 } & Positive & Negative & \\
\hline Positive & 97 & 11 & 108 \\
Negative & 10 & 266 & 276 \\
Total & 107 & 277 & 384 \\
\hline
\end{tabular}

Sensitivity $90.7 \%, 96.7 \%, 91.7 \%, 96.4 \%, \chi^{2}=291.9667 \operatorname{Pr}=0.000$

Cases of malaria was categorized using parasitic density; at parasite density greater than or equal ten thousand the case was identified as sever and when parasite density was less than ten thousand the case was categorized as mild. Using these as marker of differentiation, $27.1 \%$ of severe and $72.9 \%$ of mild malaria cases were reported. Severe malaria was increasingly observed in P.vivax $(27.5 \%)$ than P.falciparum (23.3\%) (Table 4).

Table 4. Characterization of malaria case based on parasite density at Adama Malaria Center from mid November to December 2012.

\begin{tabular}{lccc}
\hline \multirow{2}{*}{ Blood film result } & \multicolumn{2}{c}{ Case definition } & \multirow{2}{*}{ Total } \\
\cline { 2 - 3 } & Severe N (\%) & Mild N (\%) & \\
\hline P. vivax & $25(27.5)$ & $66(72.5)$ & 91 \\
P. falciparum & $4(23.3)$ & $11(64.7)$ & 17 \\
Mixed & - & $1(100)$ & 1 \\
Total & $29(27.1)$ & $78(72.9)$ & 107 \\
\hline
\end{tabular}

\section{Discussion}

The World Health Organization has recognized the urgent need for simple diagnostic tests for malaria to overcome the deficiencies of light microscopy (Moody, 1996). Their action plan for malaria control from 1995 is strengthening national capabilities to provide early diagnosis and treatment both within and outs side the health services [16]. In view of this, the study evaluated Rapid Diagnostic Test (RDT) kit the SD FK80 Bioline for the diagnosis of P.falciparum/P.vivax malaria at Adama malaria center.

The prevalence rate of malaria in this study was $30.7 \%$ which is similar with previous study conducted in Ethiopia (31\%) [11].

The present study evaluated the performance of the RDT (SD FK80) against microscopy which is gold standard. In this study, the RDT (SD FK80) sensitivity was found to be $90.7 \%$ while specificity was $96.7 \%$. Positive predictive value and Negative Predictive value were $91.7 \%$ and $96.4 \%$, respectively. Similar study done in Antwerp, Belgium, indicated consistent result in overall sensitivity of RDT (SD FK80) kit for the diagnosis of P.falciparum and P.vivax were $91.6 \%$, and specificity of $99.2 \%$ [10]. In contrast, a study conducted in Nigeria reported that the sensitivity of SD Bioline P.f/ P.v was low (47\%) while specificity was high $100 \%$ [16].

Finding of this study revealed that there were combinations of positive RDT result and negative slide findings in $10(2.3 \%)$ of the cases (probable malaria) and a negative RDT result with positive slide findings in $11(2.8 \%)$ of the patients (possible malaria).This may occur due to cross-reactivity with heterophile antibodies [17]. Moreover, presence of P.falciparum gametocytes also produces $\mathrm{pLDH}$ and this test may remain positive despite clearance of the asexual parasite forms [18]. In this study, seven mixed infections were detected by RDT and this mixed infection is could be to the presence of gametocyte in the peripheral blood which caused a different result with the microscopy.

Severe and complicated malaria is usually caused by P.falciparum than P.vivax. In contrast, in the present study 
severe malaria was increasingly observed in P.vivax $(27.5 \%)$ than P.falciparum $(23.3 \%)$. This finding is similar with previous studies from Indonesia, Papua New Guinea, Thailand and India where severe vivax malaria was reported to be $21 \%-27 \%$ [19]. However, this is by large less than from the findings of another study conducted in Brazil which indicated severe P.vivax accounted for 59.4\% [20].

Severe complications associated with vivax malaria have also been reported in the Amazon region, together with rising documentation of drug resistance worldwide, the complications of P.vivax infection represent a global health menace which needs focused efforts to its resolution [21]. In this study, higher prevalence of sever vivax malaria may be due to unknown reason since the exact pathogenesis and organ-specific morbidity caused by P.vivax infection remains unrecognized and poorly studied because of a paucity of research in this area.

Generally, high proportions of P.falciparum cases are expected in Ethiopia during the peak malaria transmission.

In contrary, this study revealed that P.vivax was the predominant species in the study area. This finding goes in agreement with the current trends shift in malaria cases occurrence as it is supported by a study conducted by Alemu et al (2012). Accordingly, the dominant species in Ethiopia was P.falciparum but since 2008 P.vivax was becoming the dominant malaria parasite in Ethiopia in general [22] and in the study area in particular. The proportions of P.vivax malaria have been low during epidemics and peak malaria transmission seasons compared to P.falciparum, as observed from the previous data in the country. The possible reason for this trend shift from $P$. falciparium to P.vivax might be due to the public health importance of P.vivax that is frequently overlooked and left in the shadow of the enormous problem caused by $P$. falciparum [23].

\section{Conclusion and Recommendations}

From this study it can be conclude that the Rapid test kit is satisfactory for diagnosis of P.falciparum and P.vivax infections. It is a useful adjunct to microscopy especially in our country where there is limited man power and resource. Besides, it was found that P.vivax is causing more of sever malria in the study area. Hence, due attention should be given to control this species in line with the existing treatment/control strategies of P.falciparum.

\section{References}

[1] Alioune B Ly, Tall A, Perry R, Baril L, Badiane A, Faye J, et.al. Use of HRP-2-based rapid diagnostic test for Plasmodium falciparum malaria: assessing accuracy and costeffectiveness in the villages of Dielmo and N diop, Senegal. . Malaria Journal. 2010; 9:153.

[2] Federal Democratic Republic of Ethiopia Ministry of Health. Ethiopian National malaria indicator survey, 2007.

[3] Abeku T. Spatial and temporal variations of malaria epidemic risk in Ethiopia: factors involved and implications. J Acta Tropica. 2003; 87: 331-340.

[4] Martens P and Hall L. Malaria on the move: Human population movement and malaria transmission. Emerg Infect Dis. $2000 ; 6: 28-45$

[5] Tulu AN. The Ecology of Malaria Health and Disease in Ethiopia. 1993; Pp.341-352.

[6] Moody A. A rapid dipstick antigen capture assay for the diagnosis of falciparum malaria. WHO Informal Consultation on Recent Advances in Diagnostic Techniques and Vaccines for Malaria. Bull World Health Organ.1996; 74:47-54.

[7] Neeru S, Man M, Mohan KS, Rajiv KM, Shweta S, et.al. Field and laboratory comparative evaluation of rapid malaria diagnostic tests versus traditiona and molecular techniques in India. J Malaria. 2010; 9:191.

[8] World Health Organization. Malaria (P. falciprum/P. vivax) W/B, RapiCard TM Insta Test Cortez Diagnostic, Inc, 2009.

[9] Faisal RM. Laboratory Diagnosis of Malaria. 2009, Third Edition.

[10] Gillet P, David PD, Emmanuel B, Lieselotte C, Marjan VE, Jan J. Test characteristics of the SD FK80 Plasmodium falciparum/Plasmodium vivax malaria rapid diagnostic test in a non-endemic setting. J Malar 2009) 8: 26.

[11] Mohammed H, Kassa M, Kebede A. Paracheck-pf® Test Versus Microscopy in the Diagnosis of Falciparum Malaria in Arbaminch Zuria Woreda of South Ethiopia. J Ethiopian Health science. 2012; 22: 93-8.

[12] World Health Organization. A comparative study of three rapid diagnostic tests (RDTs) for malaria diagnosis in Oromia Regional State, Ethiopia. World Health Organization, 2000.

[13] Bekele S, Mengistu L, Abebe A, Daddi J, Girmay M, Berhanu E. Evaluation of the performance of CareStartTM Malaria $\mathrm{Pf} / \mathrm{Pv}$ Combo and Paracheck $\mathrm{Pf}{ }^{\circledR}$ tests for the diagnosis of malaria in Wondo Genet, southern Ethiopia. J Acta Tropica. 2009; 111: 321-324.

[14] Moody A. Rapid diagnostic tests for malaria parasites. Clin Microbiol Rev. 2002; 15:66-78.

[15] Altman DG. Practical statistics for medical research. London: Chapman and Hall, 1991.

[16] Zaccheaus AJ, Emmnuel KU, Fiekumo IB, Theresa AJ. Field Evaluation of SD Bioline Rapid Malaria Diagnostic Test among Asymptomatic Malaria Infected Children in Port Hareourt Nigeria. J Parasitology. 2007; 2:39-44.

[17] Biswas S, Tomar D and Rao DN. Investigation of the kinetics of histidine-rich protein 2 and of the antibody responses to this antigen, in a group of malaria patients from India. Ann Trop Med Parasitol. 2005; 99:553-562.

[18] Chansuda W, Mazie JB, Sinuon M, Awalludin S and Walther HW. A Review of Malaria Diagnostic Tools: Microscopy and Rapid Diagnostic Test (RDT). American J of Tropical Medicine and Hygiene. 2007; 77.

[19] Price RN, Douglas NM, Anstey NM. New developments in Plasmodium vivax malaria: severe disease and the rise of chloroquine resistance. J Infect Dis. 2009. 22:430-435. 
[20] Andrade B, Reis-Filho A, Sebastião SN, Jorge C, Luis MA C, et.al. Severe Plasmodium vivax malaria exhibits marked inflammatory imbalance. J Malaria. 2010; 9:13.

[21] Daniel G. The molecular genetics of blood group polymorphism. Transpl Immunol. 2005 14:143-153.
[22] Alemu A, Muluye D, Mihret M, Adugna M, Gebeyaw M. Ten year trend analysis of malaria prevalence in Kola Diba, North Gondar,Northwest Ethiopia. Parasites. 2012; 5:173.

[23] Baird JK. Neglect of Plasmodium vivax malaria. Trends Parasitol. 2007; 23:533-539. 